\title{
eHealth Applications in Health Care Management Per-Gunnar Svensson
}

Address: Director General, International Hospital Federation, London, UK

E-mail: pergunnar@ihf.co.uk

Published: 17 September 2002

Received: 23 June 2002

EHealth International 2002, I:5

Accepted: 17 September 2002

This article is available from: http://www.ehealthinternational.org/content/I/I/5

(C) 2002 Svensson; licensee BioMed Central Ltd. This article is published in Open Access: verbatim copying and redistribution of this article are permitted in all media for any non-commercial purpose, provided this notice is preserved along with the article's original URL.

\section{Introduction}

There are seemingly an endless number of possible applications of information technology (IT) to health service management. Enthusiasm in introducing IT solutions in health care is sometimes bypassing traditional scrutiny and quality control. Without proper assessment and system thinking (how implementation, in part of the system, may sometimes produce negative effects in other parts of the system) one should not introduce new IT solutions.

The area of e-health is as said, very broad, covers topics such as telemedicine, electronic records, recruitment, going paperless, procurement, healthcare score cards, audits, information systems etc.

Detmer [1] defines three areas of health informatics:

- Consumer informatics

- Medical and clinical informatics, and

- Bio informatics.

These categories are based on the predominant type of user or use.

In this paper, the focus will be on the first two types mentioned.

\section{Consumer informatics}

Often this category - Consumer Informatics - is the one commonly referred to as 'e-health' and focuses communications to patients and the public about health topics.

Consumer-to-consumer (C-to-C) applications are potentially strong means of empowering individuals and the public. There are 25,000 - 30,000 health-oriented websites and they are among the most visited. These sites are and will be major sources of information and mis-information. There is an urgent need for all concerned, including politicians/lawmakers, health professionals and industry to put in place adequate standards and quality control for these websites.

Already C-to-C applications have contributed to the creation of "virtual" and sometimes powerful communities; sometimes with questionable outcomes, such as the violence in connection with the World Trade Organization Summits. But, sometimes, more in line with the spirit of well-informed democracy, such as networking among landowners suffering flooding in England. Both examples cited were focussing on government(s) and other concerned parties.

\section{Medical / clinical informatics}

This category relates directly to health care structure, processes and outcomes. A main application is computerbased medical records, a sub-category of which is computer-based personal records that will facilitate access to low cost therapies, for example, with certain areas of mental health, such as depression. 
Another sub-category is computer-based patient records that will facilitate clinical decision-making. These later records may be linked to knowledge-oriented systems that may contribute to quality control of clinical processes. Such a decision support has been demonstrated to have improved outcomes.

Computer-based population or community health records are usually anonymized patient and/or personal records. These systems are particularly valuable in public health where one is trying to trace different types of health hazards, linked either to medical, environmental or social agents.

What general comments, therefore, can be made regarding computer-based records? There is certainly important ethical concerns in relation to composition of records and access to the same. Also, linking different record systems to each other sometimes raises criticism, in particular in cases, which may involve personal/patient records. Again, there is need to secure standards and qualities and for appropriate steps, nationally and internationally, to be taken in the search for solutions.

Also, lack of guidance from central authorities, have in many instances led to a mish-mash of non-compatible computer-based patient record systems. Such circumstances have caused problems to arise in the smooth processing of patients between health service units, even within the same health authority (or equivalent). In spite of the many positive aspects of devolution/decentralisation, there is, as demonstrated in the above-cited examples, a need for central coordination. This observation may also have some bearing internationally with the high volume of people travelling across national boarders and sometimes needing emergency health treatment outside of their respective countries. In these cases, quick and efficient transfer, electronically, of medical records may be essential for achieving delivery of good quality acute care.

\section{Telemedicine}

Finally, telemedicine provides a category by itself. Telemedicine, meaning healthcare delivered by electronic means, has been on the road for over a century - if care provided by telegraph and telephone is considered. However, towards the end of the last century, this emerged as a delivery system with huge potential due to the information technology revolution, which made two-way, audiovisual transmission possible at reasonable cost.[2]

The views expressed by Hjelm, point to the difficulties (that can not be fairly presented here) telemedicine is facing, result in many shortcomings. It has a long way to go before it can be effectively integrated into a healthcare delivery system. One crucial difficulty is that many telemed- icine applications have yet to be developed, evaluated and implemented in the hospital environment, before application of the system over longer distances.

\section{Concluding remarks}

As mentioned, information technology and e-health have great potential. Research and development studies, however, are needed in assessing narrow and broader implications of IT applications. These cannot be left to IT enthusiasts alone, neither to less well-informed politicians or health professionals. Applications should, in my mind, be built up incrementally by starting from smaller scale pilot projects. At later stages and after careful assessment, larger scale implementations may be appropriate. Nationally and internationally, there is a need for concerted action in developing standards (in order to reach compatibility) and ethical frameworks.

From the perspective of the International Hospital Federation, we have noted, with surprise and satisfaction, that our own website, [www.hospitalmanagement.net], has reached an audience much bigger than expected. In less than a year after inception, around 550, 000 hits per month (November 2001) and some 1000 working sessions a day, have been recorded. We acknowledge, therefore, this website as a powerful tool in reaching members, potential members, industry and the public. The running of our website is dependent on advertising. Considering that many claim there is growth in health-related e-commerce as well as an increase in cost savings in the use of ecommerce facilities, we are hopeful and confident in the future development of our website.

\section{References}

I. Detmer D: Transforming Health Care in the Internet Era. World Hospitals and Health Services 2001, 37:2

2. Hjelm M: Making Telemedicine an In-patient. Hospitals International 200I, 37:2 